\title{
Overview of prebiotics, probiotics and synbiotics
}

\section{N Schellack, Y Combrinck}

\author{
School of Pharmacy, Sefako Makgatho Health Sciences University, South Africa \\ Corresponding author, email: natalie.schellack@smu.ac.za
}

Probiotics have become well-known and widely-used, especially as supplements or add-on therapy in the prevention and management of antibiotic-associated diarrhoea. Very often, however, the beneficial action and correct use of probiotics may either be seriously misunderstood or not acknowledged at all. This article provides an overview of prebiotics, probiotics and synbiotics, including their mechanisms of action, clinical applications and available products on the local market.

Republished with updates from SAPJ 2018;85(5):22-28

\section{Introduction}

The history of probiotics began in the early 1900s. ${ }^{1}$ It is hypothesised that people who used fermented milk products containing lactic acid bacteria [Streptococcus thermophilus and Lactobacillus delbrueckii (subspecies bulgaricus)] had a decreased intestinal $\mathrm{pH}$, and that the fermented milk suppressed the overgrowth of harmful bacteria. ${ }^{1}$

It is fair to assume that hygiene was difficult to establish in the early 1900s. Soldiers were severely affected by enterobacteria during World War I, in around 1917.2 The family, Enterobacteriaceae (Gram-negative rods), were found to be associated with plant material, as well as soil and water. ${ }^{2}$ This might have been owing to the difficult circumstances of that time, combined with the lack of hygiene. ${ }^{2}$ The soldiers were severely affected by shigellosis. However, German professor, Alfred Nissle, noticed that during this severe outbreak, one of the soldiers was not affected. ${ }^{2}$ Through careful research, he isolated a non-pathogenic strain of Escherichia coli from the faeces of this soldier. All indications suggested that this non-pathogenic strain had prevented the soldier from acquiring shigellosis. ${ }^{2}$ This was subsequently interpreted to be a so-called probiotic. The strain isolated by Nissle in 1917 is an example of a non-lactic acidproducing, bacterial probiotic. ${ }^{2}$

The growth of favourable organisms can be stimulated by microbial factors. The term 'probiotics' was introduced in 1965. 1,2 Relevant and commonly used terminology are described in Table 1.2,3

\section{What are probiotics?}

Probiotics are microorganisms, and contribute to the health of a host. The host may benefit from probiotics when live microorganisms are administered in an adequate amount to restore microflora symbiosis in the gastrointestinal tract. This has been acknowledged by the Food and Agriculture Organization of the United Nations and the World Health Organization. ${ }^{4}$

A high percentage of probiotics derive from Lactobacillus, Bifidobacterium, S. thermophilus and homeostatic soil organisms. These Gram-positive bacteria and strains are also found in hair, skin, the mouth, respiratory tract, intestinal tract and other parts of the human body. Favourable strains, such as L. acidophilus, L. bulgaricus, L. casei, L. plantarum, L. rhamnosus, L. salivarius, L. sporogenes, and B. bifidus, B. bifidum, B. infantis and B. longum, are used in probiotic formula. Yeast, such as Saccharomyces boulardii, is also included in probiotics. ${ }^{4}$

Probiotics that are most frequently encountered contain the Bifidobacterium and Lactobacillus spp. These organisms are the predominant and subdominant organisms of the gastrointestinal microbiota, and are also added to different types of food. The yeast species, S. boulardii, has also been shown to have a beneficial effect on health status. Food industry personnel are very interested in these organisms, because of the beneficial effects that they have on health, as well as the history of the safe use of fermented milk products. ${ }^{5}$

Table 1: General probiotic-related terms and definitions ${ }^{2,3}$

\begin{tabular}{|c|c|}
\hline \multirow[t]{2}{*}{ Lactic acid bacteria } & $\begin{array}{l}\text { Gram-positive bacteria which are fermentative, non-pathogenic and non-toxigenic, and produce lactic acid from } \\
\text { carbohydrates, which makes them valuable for food fermentation }{ }^{2}\end{array}$ \\
\hline & These species include Lactobacillus, Lactococcus and Streptococcus thermophilus ${ }^{2}$ \\
\hline Fermentation & $\begin{array}{l}\text { Microorganisms produce lactic acid, ethanol and other metabolic end-products to convert food into other } \\
\text { products }^{2}\end{array}$ \\
\hline Species & A group of related bacteria, which are highly similar by phenotype, but differ in specific characteristics ${ }^{3}$ \\
\hline Bacteriocins & Proteins that are produced by some bacteria. They constrain or kill closely related species ${ }^{3}$ \\
\hline Mucin & Glycoprotein that contains a high molecular weight and is found in the secretion of mucous membranes ${ }^{3}$ \\
\hline
\end{tabular}


Probiotics should adhere to specific standards, including their tolerance of gastric acid and bile in the gastrointestinal tract, ability to adhere to the gastrointestinal mucosa, and their competitive exclusion of pathogens. ${ }^{5}$ Probiotics should be able to adhere to cells, and should exclude or reduce pathogenic adherence. ${ }^{4}$ Probiotics must also be able to persist, multiply and produce acid. ${ }^{4}$ Probiotics need to be safe, non-invasive, noncarcinogenic and non-pathogenic, as well as being able to form normal balanced flora. ${ }^{4}$

The survival of probiotics through the gastrointestinal tract can be influenced by the acidity of the stomach, the concentration and length of exposure to the acid and bile salt, and the level of bile salt hydrolase activity. Therefore, it is important that probiotics can survive gastric and bile acid when administered so that they can reach the gastrointestinal tract. They must also be able to colonise the host epithelium, and demonstrate a beneficiary effect. It has been shown that non-spore-forming lactobacilli-type probiotics are inactive in the low gastric $\mathrm{pH}$ and the bile. Probiotics can be found in food and dietary supplements, such as tablets, capsules and powder. It has been stated that the bacteria may have already been present or added during the preparation of probiotic food. These probiotics should be stored in acceptable conditions to ensure that they have long-term activity and feasibility for use in the general population. ${ }^{4}$

\section{How do probiotics work?}

Initially, it was difficult to understand probiotics' mechanism of action. Probiotic bacteria can stimulate the host defence mechanisms by enhancing the immune system, which acts on both humeral and cellular responses. Probiotics can also ease digestion by stabilising the microflora, as well as preventing hypersensitivity-reactions to food antigens. ${ }^{1}$

In stimulating the synthesis of immunoglobulins and cytokines, the effects of general probiotics are also associated with modulation of the immune response. Lactobacillus spp. shows macrophage activation, as well as an increase of phagocytosis, as confirmed by various clinical studies. Organisms such as bacteria, fungi and viruses are responsible for activation of the inflammatory cascade. Probiotics reduce the inflammatory reaction and simultaneously enhance the immune response. The duration of acute infections, like diarrhoea in children, traveller's diarrhoea and diarrhoea caused by Clostridium difficile infection, is effectively reduced by numerous probiotic strains, including L. reuteri, L. rhamnosus and L. casei. ${ }^{1}$

It is clear that multiple factors are prominent with regard to the beneficial effects of probiotics, although the mechanisms are not yet fully understood. Mechanism of action is achieved as: ${ }^{5}$

- The gastrointestinal epithelial barrier function is enhanced

- Pathogen adhesion is inhibited, owing to concomitant probiotic adhesion to the intestinal mucosa

- Pathogenic microorganisms are excluded through competition with the probiotics

- Anti-microorganism substances are produced

- The immune system is modulated.

Immune modulation is achieved through the interaction of the probiotics and the host cells. ${ }^{6}$ The target is predominantly gastrointestinal epithelial- and gastrointestinal-associated immune cells in this process. ${ }^{6}$ An overview of naturally occurring human intestinal microbiota is provided in Table 2.2,4,7

The non-immune mechanisms and the mucosal immune mechanisms show a positive reaction when stimulated by probiotics, affecting the intestinal ecosystem. This is achieved through antagonism and competition with potential pathogens. Probiotics are mostly recognised for the decrease in the incidence of diarrhoea, as well as the severity of the disorder. Excellent results have been obtained in certain animal models in decreasing colon cancer, probably due to the suppressing activity of certain bacterial enzymes which may have raised the levels of the procarcinogens. Unfortunately, this has not yet been achieved in human models. Probiotics have numerous

Table 2: An overview of human intestinal microbiota ${ }^{2,4,7}$

\begin{tabular}{|c|c|}
\hline Oral cavity (saliva) ${ }^{4}$ & $\begin{array}{l}\text { Bacteroides, Bifidobacterium, Corynebacterium, Fusobacterium, Lactobacillus, Neisseria, } \\
\text { Staphylococcus, Streptococcus, Veillonella and yeast }{ }^{4,7}\end{array}$ \\
\hline \multirow{6}{*}{$\begin{array}{l}\text { Stomach }(\mathrm{pH} \text { of } 1-2) \text { and duodenum } \\
(\mathrm{pH} \text { of } 6-7)^{4}\end{array}$} & Only a small number of microorganisms, because of the low $\mathrm{pH}^{7}$ \\
\hline & $<10^{3}$ bacterial cells per gram of stomach contents ${ }^{2,7}$ \\
\hline & Mainly bacilli, enterococci, Helicobacter, lactobacilli and streptococci $2{ }^{2,7}$ \\
\hline & Acidic, pancreatic secretions and bile secretions cause an unfavourable environment for microbes ${ }^{2,7}$ \\
\hline & Stomach: Bacteroides, Bifidobacterium, Enterobacteriaceae, Lactobacillus, Streptococcus and yeast ${ }^{4}$ \\
\hline & $\begin{array}{l}\text { Duodenum: Bacteroides, Bifidobacterium, Enterobacteriaceae, Lactobacillus, Streptococcus, Veillonella and } \\
\text { yeast }^{4}\end{array}$ \\
\hline \multirow[t]{2}{*}{ Jejunum and ileum ( $\mathrm{pH}$ of $6-7)^{4}$} & There is a progressive increase in the number and diversity of the bacteria ${ }^{2,7}$ \\
\hline & Ileum: Bacteroides, Bifidobacterium, Clostridium, Enterobacteriaceae, Lactobacillus, Streptococcus and yeast ${ }^{4}$ \\
\hline \multirow[t]{3}{*}{ Colon $(\mathrm{pH} \text { of } 5-7)^{4}$} & The large intestine contains a high population of anaerobes ${ }^{2}$ \\
\hline & The colon contains the majority of the regular gastrointestinal microbes ${ }^{7}$ \\
\hline & $\begin{array}{l}\text { Colon: Bacteroides, Bifidobacterium, Clostridium, Coprococcus, Enterobacteriaceae, Eubacterium, Lactobacillus, } \\
\text { Peptostreptococcus, Ruminococcus and Streptococcus }{ }^{4}\end{array}$ \\
\hline Faeces $^{4}$ & $\begin{array}{l}\text { Faeces: Bacteroides, Bifidobacterium, Clostridium, Coprococcus, Enterobacteriaceae, Eubacterium, } \\
\text { Lactobacillus, Peptostreptococcus, Ruminococcus, Streptococcus, Veillonella and yeast }{ }^{4}\end{array}$ \\
\hline
\end{tabular}


Table 3: The clinical applications of probiotics ${ }^{2}$

\begin{tabular}{|c|c|}
\hline Probiotic or prebiotic & Recommended dosage \\
\hline \multicolumn{2}{|l|}{ Acute diarrhoea in adults } \\
\hline Enterococcus faecium & $10^{8} \mathrm{cfu}$, three times daily \\
\hline Lactobacillus paracasei or Lactobacillus rhamnosus & $10^{9} \mathrm{cfu}$, twice daily \\
\hline Saccharomyces boulardii, a strain of Saccharomyces cerevisiae & $10^{9}$ cfu per capsule of $250 \mathrm{mg}, 2-6$ capsules per day \\
\hline \multicolumn{2}{|l|}{ Acute infectious diarrhoea } \\
\hline Lactobacillus rhamnosus & $10^{10}$ to $10^{11} \mathrm{cfu}$, twice daily \\
\hline Saccharomyces boulardii, a strain of Saccharomyces cerevisiae & $200 \mathrm{mg}$, three times daily \\
\hline \multicolumn{2}{|l|}{ Antibiotic-associated diarrhoea } \\
\hline Saccharomyces boulardii, a strain of Saccharomyces cerevisiae & 250 mg, twice daily \\
\hline Lactobacillus rhamnosus & $10^{10} \mathrm{cfu}$, once or twice daily, or even $2 \times 10^{10} \mathrm{cfu}$, twice daily \\
\hline Enterococcus faecium & $10^{8} \mathrm{cfu}$, twice daily \\
\hline Lactobacillus casei in fermented milk & $10^{10} \mathrm{cfu}$, twice daily \\
\hline Bacillus clausii (Enterogermina strains) & $2 \times 10^{9}$ spores, three times daily \\
\hline Lactobacillus acidophilus PLUS Lactobacillus casei & $5 \times 10^{10} \mathrm{cfu}$, once or twice daily \\
\hline \multicolumn{2}{|l|}{ Clostridium difficile diarrhoea in adults } \\
\hline Lactobacillus casei in fermented milk & $10^{10} \mathrm{cfu}$, twice daily \\
\hline Lactobacillus acidophilus PLUS Bifidobacterium bifidum (Cultech strains) & $2 \times 10^{10}$ cfu for each strain, once daily \\
\hline Oligofructose & 4 grams, three times per day \\
\hline Lactobacillus rhamnosus PLUS Lactobacillus acidophilus & 109 cfu each, once daily \\
\hline \multicolumn{2}{|l|}{ Helicobacter pylori eradication } \\
\hline Lactobacillus casei in fermented milk & $10^{10}$ to $10^{12}$ cfu daily, for 14 days \\
\hline Lactobacillus rhamnosus GG & $6 \times 10^{9} \mathrm{cfu}$, twice daily \\
\hline Bacillus clausii (Enterogermina strains) & $2 \times 10^{9}$ spores, three times daily \\
\hline Saccharomyces boulardii, a strain of Saccharomyces cerevisiae & $500 \mathrm{mg}$ to $1 \mathrm{~g}$, or $2-4 \times 10^{9} \mathrm{cfu}$ per day \\
\hline Kefir & $250 \mathrm{ml}$, twice daily \\
\hline Lactobacillus reuteri & $10^{8} \mathrm{cfu} /$ day \\
\hline \multicolumn{2}{|l|}{ Nosocomial diarrhoea } \\
\hline Lactobacillus rhamnosus & $10^{10}$ to $10^{11} \mathrm{cfu}$, twice daily \\
\hline Bifidobacterium lactis PLUS Streptococcus thermophilus & $10^{8}$ plus $10^{7} \mathrm{cfu} / \mathrm{g}$ of formula \\
\hline \multicolumn{2}{|l|}{ The prevention of respiratory tract infections in athletes } \\
\hline Lactobacillus casei (Shirota strain in fermented milk) & $10^{10} \mathrm{cfu}$, once daily \\
\hline \multicolumn{2}{|l|}{ Remission in ulcerative colitis } \\
\hline Escherichia coli & $5 \times 10^{10}$ viable bacteria, twice daily \\
\hline \multicolumn{2}{|l|}{ Symptoms of irritable bowel syndrome } \\
\hline Bifidobacterium infantis & $10^{8} \mathrm{cfu}$, once daily \\
\hline Bifidobacterium animalis in fermented milk & $10^{10} \mathrm{cfu}$, twice daily \\
\hline Lactobacillus acidophilus & $10^{10} \mathrm{cfu}$, per day \\
\hline \multicolumn{2}{|l|}{ Treatment of constipation } \\
\hline Lactulose & $20-40 \mathrm{~g} /$ day \\
\hline Oligofructose & $>20 \mathrm{~g} /$ day \\
\hline \multicolumn{2}{|l|}{ Treatment of hepatic encephalopathy } \\
\hline Lactulose & $45-90 \mathrm{~g} / \mathrm{day}$ \\
\hline \multicolumn{2}{|l|}{ Treatment of mildly active ulcerative colitis or pouchitis } \\
\hline $\begin{array}{l}\text { Mixture of eight strains (one Streptococcus thermophilus, four Lactobacillus } \\
\text { and three Bifidobacterium) }\end{array}$ & $2 \times 10^{11}$ cfu each, twice daily \\
\hline
\end{tabular}

cfu - colony-forming units 
Table 4: Examples of commercially-available probiotic, prebiotic and synbiotic products in South Africa ${ }^{8}$

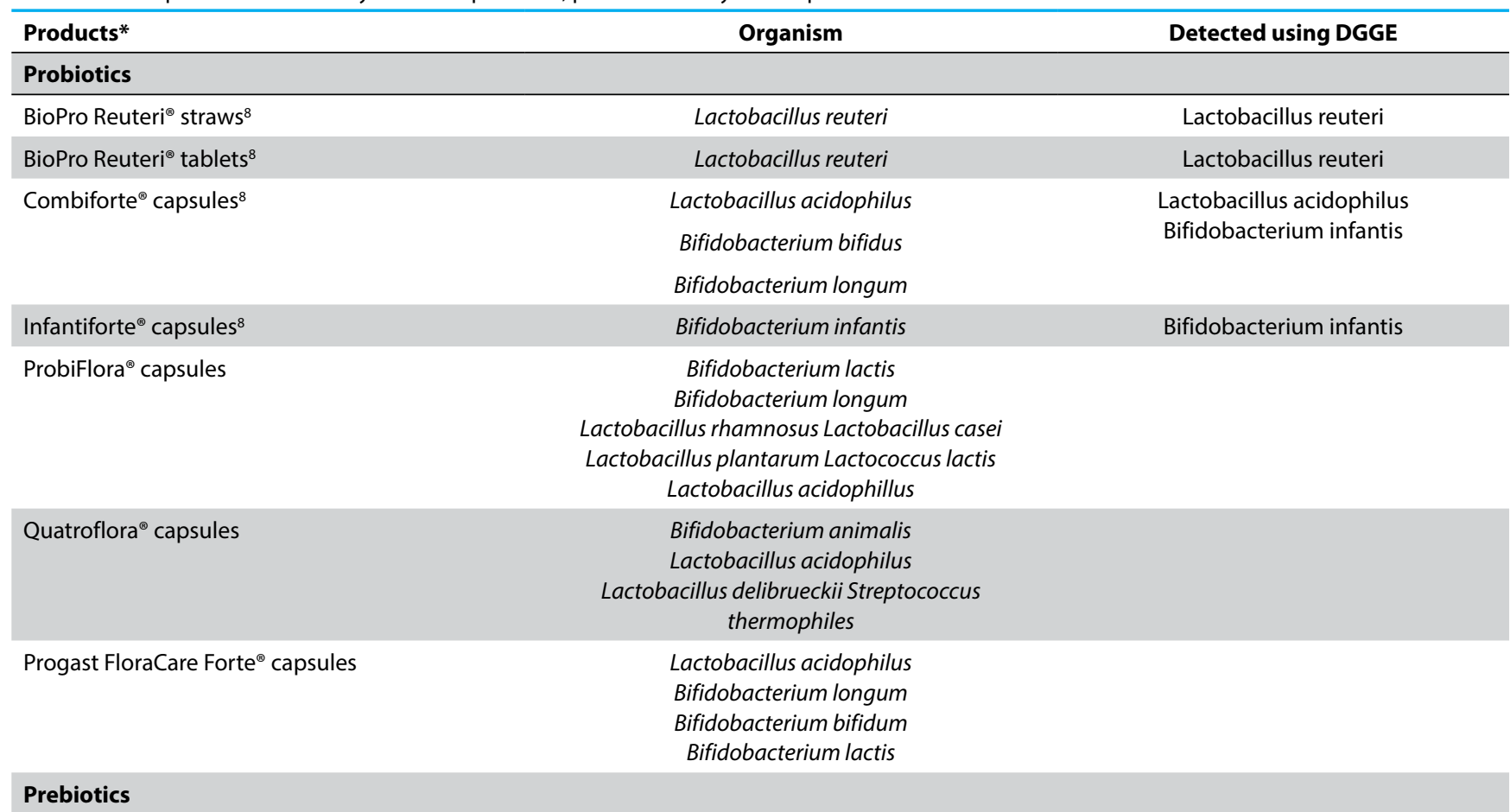

Asparagus, bananas, chicory, garlic, leeks, oats, onions, soybeans and wheat ${ }^{1}$

Synbiotics

Yoghurt and kefir ${ }^{4}$

${ }^{*}$ Other probiotic products include, Reuterina ${ }^{\oplus}$, Viral Guard ${ }^{\oplus}$ and Duphalac ${ }^{\circledast}$; DGGE - denaturing gradient gel electrophoresis

benefits, which can be classified as either beneficial or nonimmunological. ${ }^{2}$

Probiotics have the following immunological benefits: ${ }^{2}$

- Increased antigen presentation of B lymphocytes and increased secretory immunoglobulin A production is activated by the local macrophages, and affect the system both locally and systemically.

- The cytokine profiles are modulated.

- Hypo-responsiveness to food antigens is established.

Non-immunological benefits include: ${ }^{2}$

- Food digestion is improved, and increased competition with pathogens for the nutrients is achieved.

- The local $\mathrm{pH}$ is adjusted to create an unfavourable local environment for pathogens.

- Pathogens are inhibited by the production of bacterocins

- Superoxide radicals are removed.

- The epithelial mucin production is stimulated.

- The intestinal barrier function is enhanced.

- There is competition for adhesion with the pathogens.

- The pathogen-derived toxins are modified. ${ }^{2}$

Excellent results have been reported in various human studies and animal models with regard to the clinical potential of probiotics against many diseases. Probiotics have been reported to: ${ }^{5}$
- suppress diarrhoea,

- alleviate postoperative complications and lactose intolerance,

- exhibit anti-colorectal cancer and antimicrobial activity,

- reduce irritable bowel symptoms, and

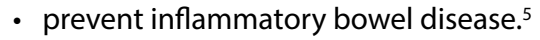

To summarise, it can be stated that probiotics are confirmed to be safe, and should adhere to certain conditions. For example, probiotics should:

- $\quad$ Not lose their properties during storage.

- Normally be present in the human intestines.

- Be able to survive in the gastrointestinal tract and colonise the intestinal cells.

- Have beneficial effects on human health.

- Display antagonism against pathogenic microorganisms.

- Not demonstrate any noticeable side-effects. ${ }^{1}$

The potential health benefits of probiotics tend to be strainspecific and generalisations of probiotic benefits do not attribute to different strains within one species. ${ }^{5}$

\section{The use of prebiotics and probiotics}

Evidence has been demonstrated in some studies, suggesting that probiotics have various clinical applications (see Table 3 ). ${ }^{2}$

\section{Prebiotics}

The term 'prebiotic' was first introduced in $1995 .{ }^{4}$ Probiotics must not be confused with prebiotics. ${ }^{4}$ Prebiotics may be described as 
a non-digestible food ingredient. ${ }^{1}$ They enhance the growth and activity of selected intestinal strains, and can therefore effect a favourable change in the balance of the intestinal microflora. ${ }^{1,7}$ Prebiotics are mainly dietary fibre, particularly soluble fibre, and are also known as 'colonic food', consisting of specific carbohydrates. ${ }^{1}$

The mechanism of action of prebiotics constitutes their effects on the intestinal bacteria through their ability to enhance the amount of beneficial anaerobic bacteria, and to decrease the pathogenic microorganism population in number. ${ }^{1,2}$

Prebiotics are present in numerous edible plants, such as asparagus, bananas, chicory, garlic, leeks, oats, onions, soy beans and wheat. Raw vegetable matter is also a key component of a high percentage of commercial prebiotics. Production is achieved via an enzymatic method, through the trans-glycosylation of monosaccharides or disaccharides, or the hydrolysis of complex polysaccharides. $^{1}$

\section{Synbiotics}

A synbiotic is a nutritional supplement containing both probiotics and prebiotics." Synbiotics can be defined as "a mixture of probiotics and prebiotics that beneficially affects the host by improving the survival and implantation of live microbial dietary supplements in the gastrointestinal tract, by selectively stimulating the growth and/or activating the metabolism of one or a limited number of health-promoting bacteria, and thus improving host welfare".

This mixture of probiotics and prebiotics works together to ensure that bacterial microflora in the gastrointestinal tract remain healthy. Synbiotic products include fermented milk products, such as yoghurt and kefir. This may be regarded as functional food, because it restores the normal bacterial microflora and supplies the necessary food for the normal microflora to proliferate. Bifidobacteria and fructo-oligosaccharides, Lactobacillus GG, inulin, as well as bifidobacteria and lactobacilli with fructo-oligosaccharides or inulin, are the best combinations of available synbiotics. ${ }^{4}$ Some of the priobiotic formulations for example Progast FloraCare Forte ${ }^{\circledR}$ Capsules is in a novel acid resistant capsules that can deliver efficient targeted delivery.

An overview of commercially-available probiotic, prebiotic and synbiotic products is provided in Table 4.

\section{Conclusion}

Probiotics are live non-pathogenic microorganisms, which have a beneficial effect on the health of the host. They are present in the gastrointestinal tract without causing any side-effects. Probiotics can be used for several conditions, e.g. antibioticinduced diarrhoea, irritable bowel syndrome and inflammatory bowel disease. Prebiotics are known to be a non-digestible food ingredient. They exert a favourable change in the balance of intestinal microflora by enhancing the growth and activity of some intestinal strains. Synbiotics, a combination of probiotics and prebiotics, are a nutritional supplement.

\section{References}

1. Szkaradkiewicz AK, Karpinski TM. Probiotics and prebiotics. Journal of Biology and Earth Sciences. 2013;3(1):M42-M47.

2. Guarner F, Khan AG, Garisch J, et al. Probiotics and prebiotics: global guidelines. World Gastroenterology Organisation, 2011.

3. Van Demark PJ, Batzing BL. The microbes: an introduction to their nature and importance. Menlo Park: Benjamin/Cummings Publications, 1987.

4. Iannitti T, Palmieri B. Therapeutical use of probiotic formulations in clinical practice. Clin Nut. 2010;29(6):701-725. https://doi.org/10.1016/j. clnu.2010.05.004

5. Bermúdez-Brito M, Plaza-Díaz J, Muñoz-Quezada S, Gómez-Llorente C, Gil A Probiotic mechanisms of action. Annal Nutr Metab. 2012;61(2):160-174. https:// doi.org/10.1159/000342079.

6. Oelschlaeger TA. Mechanisms of probiotic actions: a review. Int J Med Microbiol. 2010;300(1):57-62. https://doi.org/10.1016/j.jimm.2009.08.005.

7. Binns N. Probiotics, prebiotics and the gut microbiota. Washington DC: International Life Sciences Institute, 2013.

8. Elliott E, Teversham K. An evaluation of nine probiotics available in South Africa, August 2003. S Afr Med J. 2004;94(2):121-124.

9. Kochan P, Chmielarczyk A, Szymaniak L, et al. Lactobacillus rhamnosus administration causes sepsis in a cardiosurgical patient: is the time right to revise probiotic safety guidelines? Clin Microbiol Infect. 2011;17(10):1589-1592. https:// doi.org/10.1016/j.jimm.2009.08.005.

10. Muszynski Z, Mirska I, Matuska K. Rods of Lactobacillus genus: factor of opportunistic infections in children [in Polish]. Przegl Epidemiol. 2007;61(1):79-84 